\title{
MOLUSCOS DEL TIRRENIENSE (PLEISTOCENO SUPERIOR) DE LA PLAYA LA ARAÑA-CALA DEL MORAL (MÁLAGA)
}

Trabajo presentado a las XVIII Jornadas de Paleontología y

II Congreso Ibérico de Paleontología

Universidad de Salamanca

Salamanca, 24-29 de Septiembre de 2002

\author{
José Luis VERA-PELÁEZI, M. Carmen LOZANO- \\ FRANCISCO ${ }^{l}$, Julián RAMOS FERNÁNDEZ ${ }^{2}$ y \\ Miguel CORTÉS SÁNCHEZ

\footnotetext{
${ }^{1}$ Museo Municipal de Paleontología. C/ Matías Prats, s/n. Plaza de Toros. 29680 Estepona (Málaga). E-mail: delcultu@teleline.es

2 Guillermo Carrera Rubio, 6, 10-izq, 29004 Málaga. E-mail: julianramos@supercable.es

${ }^{3}$ Área de Prehistoria. Universidad de Córdoba. Plaza Cardenal Salazar, s/n, 14071-Córdoba. e-mail: mm.cosi@teleline.es
}

Vera-Peláez, J. L., Lozano-Francisco, M. C., Ramos Fernández, J. y Cortés Sánchez, M. 2004. Moluscos del Tirreniense (Pleistoceno Superior) de la Playa la Araña-Cala del Moral (Málaga). [Mollucs from Tyrrhenian (Upper Pleistocene) of the Beach of Araña-Cala del Moral (Málaga).] Revista Española de Paleontología, 19 (2), 251-259. ISSN 0213-6937.

\begin{abstract}
A taxonomic study of the molluscs of Tyrrhenian (Upper Pleistocene) of Araña-Cala del Moral beach (Málaga) is accomplished. A list of 45 species of invertebrates: 43 molluscs, one Echinodermata (echinids) and one choral (Antozoa) have been identified. This is considered the second outcrop with most biodiversity of Andalucian Tyrrhenian after Roquetas de Mar (Almería).
\end{abstract}

Keywords: Molluscs, Tyrrhenian, Upper Pleistocene, Beach of Araña-Cala del Moral, Málaga

\section{RESUMEN}

Se ha realizado un estudio taxonómico de los moluscos del Tirreniense (Pleistoceno Superior) encontrados en la Playa de la Araña-Cala del Moral (Málaga). Se aporta un listado de 45 especies de invertebrados, de los que 43 son moluscos, un equinodermo (Echinoidea) y un coral (Antozoa). Éste constituye el segundo afloramiento con mayor riqueza específica del Tirreniense andaluz después de Roquetas de Mar (Almería).

Palabras clave: Moluscos, Tirreniense, Pleistoceno Superior, Playa de la Araña-Cala del Moral, Málaga.

\section{INTRODUCCIÓN}

Con motivo de la solicitud de desafección de la playa fósil (Pleistoceno Superior: Tirreniense) en terrenos de la Cantera de la Araña (Málaga) se ha realizado la valoración del interés paleontológico que presenta el área comprendida al Sur, por el Mar Mediterráneo, al Este por el Cantal del Rincón de la Victoria, al Oeste por el Cerro del Candado y Almellones y al Norte, hasta Cerro Juan (Fig. 1). Este estudio queda enmarcado dentro del Convenio establecido entre la Universidad de Málaga y la Sociedad Financiera y Minera S.A. denominado "Inventario, Catalogación y Valoración de los Bienes Culturales (Arqueológicos, Geomorfológicos y Paisajísticos) en el territorio afectado por las canteras y la
Fábrica de Cemento de la empresa Financiera y Minera S.A. en el paraje de la Araña" (Ferre, 2001).

Por otro lado, también se adjuntan los datos sobre datación que se pueden obtener a partir de los estudios de la fauna, teniendo en cuenta que desde un punto de vista paleontológico sólo podemos referirnos a aquellos niveles en los que se hallaron moluscos. Para la datación y valoración de otros niveles se debe recurrir a los informes geológicos y geomorfológicos. El informe geológico nos indica que las distintas cotas estudiadas se corresponden con playas o acantilados fósiles relacionados con variaciones eustáticas. Sólo con la visión de conjunto es posible interpretar estas variaciones eustáticas, así como la geología y la paleontología de la zona (Ferre, 2001). 
En el Complejo del Humo se detectó dentro de una de las cuevas, un importante nivel de malacofauna marina correspondiente a la cota de 4-5 m sobre el nivel del mar (m s.n.m.) con abundantísima fauna de la zona infralitoral somera junto a unos niveles arqueológicos muy interesantes, con abundante malacofauna, especialmente de mejillones [Mytilus edulis (Linné, 1758)] y lapas (patéllidos) con evidencias de acción antrópica, que se encuentra en la actualidad en estudio.

Figura 1. a, Localización geográfica del yacimiento de La Araña (Málaga, S España). b, Mapa geomorfológico del área en estudio indicando los puntos de muestreo. El círculo corresponde a la cota 0,90-1,20 m s.n.m.; el cuadrado a 10-12 m s.n.m. y el triángulo a 30-35 m s.n.m. [Modificado de Azema (1961, 1995)]. a, Geographic location of outcrop LaAraña (Malaga, S. Spain). b, Geomorphology map with the main outcrops. The circle consists of 0.90-1.20 m on sea level; the square to $10-12 \mathrm{~m}$ on sea level; and the triangle to 30-35 $\mathrm{m}$ on sea level. [Modified from Azema (1961, 1995)].
Sólo en las cotas de 0.90-1.20 m s.n.m., en las estaciones o punto 11 (Peñón del Cuervo), punto 52 (Peñón del Cuervo), punto 48 (Playa de la Araña) y punto 46 (Punta Paloma); en la cota 10-11 m s.n.m., en la estación o punto 16 (túnel de la antigua carretera del Peñón del Cuervo) y 30-35 m s.n.m., se ha recolectado fauna fósil correspondiente al Tirreniense por la presencia de Strombus bubonius (Defrance, 1825) y fauna asociada, que es correlacionable con el Tirreniense T-II del nivel infe-
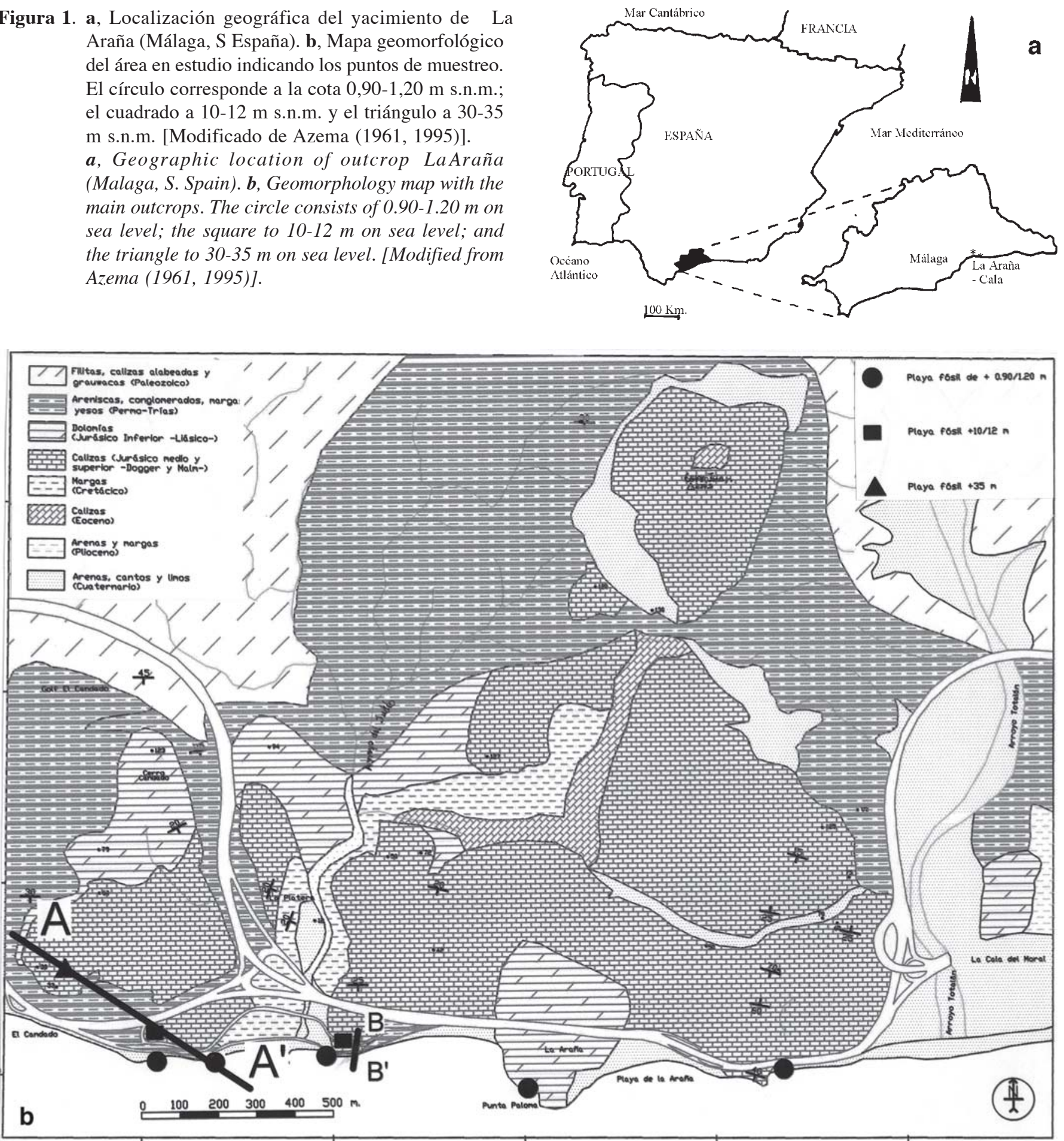
rior del Faro de Roquetas de Mar (Almería), datación sugerida por datos isotópicos (Lario et al., 1999b) para el caso de 0,90-1,20 m s.n.m. (entre 90.000 y 100.000 años). Para el caso de la cota 10-11 m s.n.m. no se puede llegar a conclusiones sobre su cronología debido a que la fauna encontrada presenta una amplia distribución cronoestratigráfica [Glycymeris insubrica (Brocchi, 1814)]: Mioceno Superior-actualidad.

\section{GEOLOGÍA}

El territorio estudiado se localiza en la costa oriental del término municipal de Málaga, entre el Arroyo Almellones, actualmente ocupado por el Campo de Golf de El Candado, al oeste, y el Arroyo de Totalán, en el límite del término del Rincón de la Victoria, al este. Por el sur limita con el Mar Mediterráneo y por el norte se ha estudiado hasta la ladera septentrional del Cerro Juan. El territorio así delimitado ocupa una superficie aproximada de 475 hectáreas (Figs. 1a, b). Pertenece a las Zonas Internas de las Cordilleras Béticas, incluidas en el denominado Complejo Maláguide (Blumental, 1927; Durand-Delga, 1968), que es uno de los conjuntos estructurales desplazados que constituyen los mantos de corrimiento que conforman el edificio de la Cordillera Bética.
Los materiales del territorio estudiado pertenecen a la "cobertera" del Complejo Maláguide. Su serie estratigráfica fue establecida por Azema (1961) y posteriormente ha sido retocada por Serrano et al. (1995) (Figs. 2 y 3).

Las dataciones absolutas procedentes del contexto occidental de la Bahía de Málaga son todavía muy escasas e impiden articular la edad de los distintos registros. Así, sólo disponemos de los siguientes datos cronológicos:

- Cueva del Tesoro: espeleotema 118.000 $\pm 20 \%$ before present (B.P.) (Durán y Soria, 1989). Costra sobre sedimentos marinos: $142.000 \pm 20 \%$ B.P., por tanto la plataforma de erosión del exterior de la cavidad es anterior al Pleistoceno Superior (Durán y Soria, 1989).

- Cueva del Humo: espeleotema que sella el tramo inferior de la columna estratigráfica: $5.700 \pm 7 \%$ B. P. y un espeleotema a la entrada de la Cueva: $21.300 \pm 7 \%$ B. P. (Ramos y Durán, 1998).

- Abrigo 3 del Complejo del Humo: datación sobre hueso (procedencia de un estrato arqueológico) por ESR: $47.300 \pm 20 \%$ B. P. (comunicación personal J. J. Durán).

- Zona del Cuervo: espeleotema cubriendo la playa fósil situada a $+0,90-1,20$ m s.n.m., con una fecha de ESR de $17.300 \pm 20 \%$ B. P. (Brükner y Ratke, 1986). Una costra similar, que también cubría el nivel marino a $+0,90$ - 1,20 m s.n.m. y/o asímismo una fecha de 90.000-100.000 B. P. (Lario et al., 1999b).

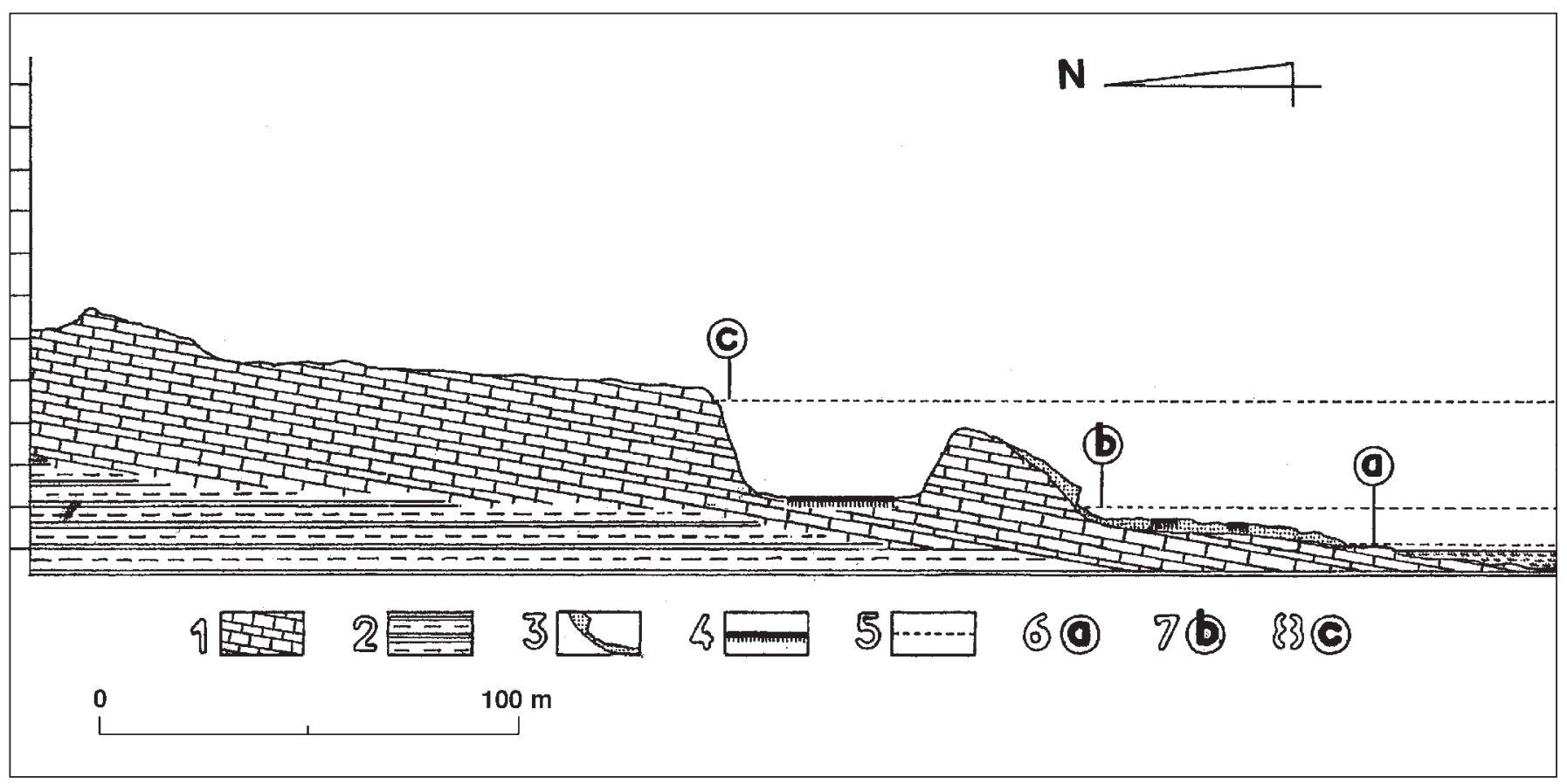

Figura 2. Corte de la sección A-A' de la figura 1b: 1, calizas jurasicas; 2, areniscas del Permo-Trías; 3, costras de ladera; 4, firme actual de la red viaria; 5, paleonivel marino; 6, nivel de playa fósil +0,90 m s.n.m.; 7, nivel de playa fósil $+10-11 \mathrm{~m}$ s.n.m.; 8 , nivel de playa fósil +35 m s.n.m.

Stratigraphic cut of the section A-A' in figure 1b: 1, Jurassic limestones; 2, Sands of Permic-Triassic; 3, Crust of hillside; 4, New highway; 5, Marin paleolevel ; 6, Fossil beach level +0.90 meters on sea level.; 7, Fossil beach level $+10-11 \mathrm{~m}$ on sea level; 8, Fossil beach level +35 m on sea level. 
- Depósitos a +10-12 m s.n.m.: Dos episodios marinos: $360.000 \pm 41.000$ y $240.000 \pm 5.000$ B.P. (U/Th) (Lario et al., 1999b).

Las playas fósiles de 0,90-1,20 m s.n.m. están constituidas fundamentalmente por gravas, arenas y una matriz arcillosa rojiza o grisácea-rojiza muy cementada con abundante malacofauna. Los moluscos se encuentran muy erosionados por el embate de las olas y fuertemente carbonatados. Este nivel se encuentra rellenando oquedades en calizas jurásicas o extendido sobre la roca de sustrato. Por encima del estrato tirreniense 0,90-1,20, aparece un nivel continental de arcillas rojas poco cementadas separadas por una clara discordancia y que se caracterizan a nivel paleontológico por la presencia del gasterópodo pulmonado Iberus alonensis (Férussac, 1821), de edad más moderna. Por encima de estos sedimentos rojizos de contacto con la playa fósil, la fauna marina desaparece, mientras que se incrementa la abundancia de Iberus alonensis.
De idénticas características son los depósitos del nivel 1011 m s.n.m., pero con los bioclastos más erosionados y una menor riqueza específica. Finalmente, en el nivel 3035 m.s.n.m., la presencia de moluscos es casi nula, con la única identificación de un fragmento de Aequipecten cf. opercularis (Linné, 1758), el grado de conservación en esta cota es malo.

\section{MATERIAL Y MÉTODOS}

Para el análisis paleontológico se han realizado cinco visitas al área en estudio. En las dos primeras se ha realizado un primer reconocimiento de las playas fósiles citadas con anterioridad en la bibliografía siendo muestreadas las que se sitúan a cotas $0,90-1,20,4-5,10-11,25-30,30-35$ y $60-65$ m s.n.m. En el presente estudio se encontró fauna en los niveles correspondientes a los situados entre $0,90-1,20,10-11$ y $30-35 \mathrm{~m}$

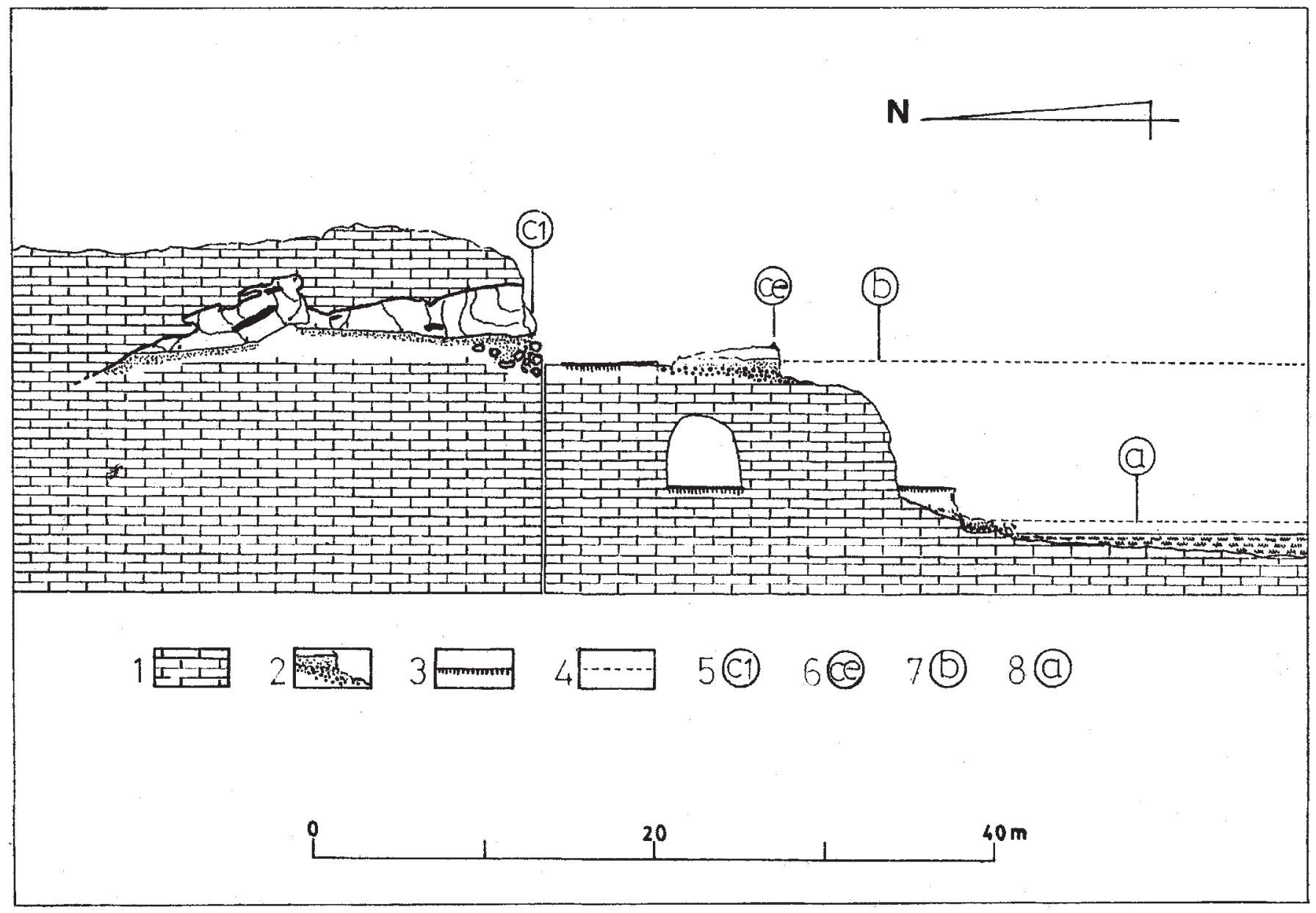

Figura 3. Corte de la sección de la Caseta del Guardia-Cuervo I (B-B') de la figura 1b: 1, calizas jurasicas; 2, sedimentos marinos cubiertos por continentales; 3 , firme actual de la red viaria; 4, paleonivel marino; 5, entrada Cueva del Cuervo I; 6, cono estalagmítico; 7, nivel de playa fósil +10-11 m s.n.m.; 8, nivel de playa fósil +0,90 m s.n.m.

Stratigraphic cut of the section B-B' (Caseta del Guardia-Cuervo I) in figure 1b: 1, Jurassic limestones; 2, Marins sediments covered by continental sediments; 3, New highway; 4, Marin paleolevel; 5, Exit of Cueva del Cuervo; 6, Stalagmitic cone; 7, Fossil beach level $+10-11 \mathrm{~m}$ on sea level; 8, fossil beach level $+0,90 \mathrm{~m}$ on sea level. 
s.n.m. Estos niveles se encuentran cubiertos por una costra de material continental rocoso y que en el caso de las cotas 4-5 y 10-11 m s.n.m. presentan fauna continental cuaternaria. Luego se realizó un primer muestreo en cada uno de los puntos visitados para detectar la posible presencia de macro o microfauna. Posteriormente, se recogió material en los puntos donde se detectó presencia de fauna fósil y se realizó un reconocimiento e identificación de la fauna in situ, con objeto de deteriorar lo mínimo posible los acantilados fósiles. Con los datos obtenidos, se ha elaborado un listado preliminar de la fauna que nos permite valorar la riqueza específica.

El número de especies identificadas, la sistemática y las conclusiones obtenidas se presentan en la tabla 1 .

\section{RESULTADOS}

Con anterioridad al presente trabajo se conocía la existencia de doce taxones en depósitos entre 0,9-1,2 m s.n.m. (Lario et al., 1999a), de los cuales Cantharus viverratus (Linné, 1758) y Strombus bubonius (Defrance, 1825) son indicadores bioestratigráficos del Tirreniense. De estas especies la primera no ha sido identificada entre el material recolectado para el presente estudio. El trabajo de campo ha permito ampliar hasta 45 los taxones asociados a estos depósitos, de los cuales 42 son primera cita para el área (Tabla 1). De las 45 especies, 43 son moluscos (27 de gasterópodos y 16 de bivalvos), espículas de un equínido regular (Echinoideo) y un par de fragmentos de un coral solitario (Antozoa).

Todos los taxones proceden de la franja infralitoral costera, excepto un elemento intrusivo procedente de los niveles continentales superiores (Iberus alonensis Férussac, 1821).

Como resultado de las prospecciones se observa que los depósitos con registros paleontológicos quedan restringidos a las cotas de 0,9-1,2, 10-15 y 30-35 m s.n.m. La presencia de Strombus bubonius y fauna asociada, permite correlacionar los ubicados a menor cota con el Tirreniense II (110.000-117.000 años B.P.) del nivel inferior del faro de Roquetas de Mar (Almería), correlación avalada asimismo por dataciones isotópicas (Zazo et al., 1981; Lario et al., 1999a); en el caso los hallazgos a 10-15 m s.n.m. no puede abordarse una datación precisa pues la fauna encontrada muestra una amplia distribución cronoestratigráfica [Glycymeris insubrica (Brocchi, 1814); Mioceno-actualidad)]. Entre 4-5 m s.n.m. existe un depósito de fauna marina que no fue posible muestrear al tratarse de un yacimiento arqueológico protegido.

Anteriormente a este estudio han sido citadas las siguientes especies en la cota 0,90-1,20 m.s.n.m. en los depósitos tirrenienses del Peñón del Cuervo-La Cala: Strombus bubonius (Defrance, 1825), Cantharus viverratus (Linné, 1758), Glycymeris insubrica (Brocchi, 1814), Acanthocardia tuberculata (Linné, 1758), Columbella rustica (Linné, 1758), Nassarius sp., Stramonita haemas- toma (Linné, 1758), Charonia lampas lampas (Linné, 1758), Patella caerulea Linné, 1758, Patella ferruginea Gmelin,1791, Mytilus edulis (Linné,1758), Balanus sp. y Ostrea sp. De este listado, Cantharus viverratus y Strombus bubonius son indicadores bioestratigráficos de Tirreniense.

Para la elaboración de la sistemática de moluscos se siguió la clasificación propuesta por Sabelli et al. (1990) para el Mediterráneo. Las especies encontradas son las siguientes:

FILUM MOLLUSCA Linné, 1758

CLASE BIVALVIA Linné, 1758

SUBCLASE PTERIOMORPHIA Beurlen, 1944

ORDEN ARCOIDA Stoliczka, 1871

Superfamilia Arcoidea Lamarck, 1818

Familia Noetiidae Stewart, 1930

Striarca lactea (Linné, 1758)

Superfamilia Limopsoidea Dall, 1895

Familia Glycymerididae Newton, 1922

Glycymeris (Glycymeris) bimaculata (Poli, 1795)

Glycymeris (Glycymeris) insubrica (Brocchi, 1814)

Glycymeris (Glycymeris) pilosa (Linné, 1767)

Glycymeris (Glycymeris) sp.

ORDEN OSTREOIDA Férussac, 1822

SUBORDEN OSTREINA Férussac, 1822

Superfamilia Ostreoidea Rafinesque, 1815

Familia Gryphaeidae Vyalov, 1936

Neopycnodonte cochlear (Poli, 1795)

SUBORDEN PECTININA Vaught, 1989

Superfamilia Pectinoidea Rafinesque, 1815

Familia Pectinidae Rafinesque, 1815

Aequipecten (Aequipecten) cf. opercularis (Linné, 1758)

Pecten (Pecten) maximus (Linné, 1758)

SUBCLASE HETERODONTA Neumayr, 1884

ORDEN VENEROIDA Adams \& Adams, 1857

Superfamilia Lucinoidea Fleming, 1828

Familia Lucinidae Fleming, 1828

Ctena (Ctena) decussata (Da Costa, 1843)

Superfamilia Carditoidea Fleming, 1828

Familia Carditidae Fleming, 1828

Cardita (Cardita) calyculata (Linné, 1758)

Superfamilia Cardioidea Lamarck, 1809

Familia Cardiidae Lamarck, 1809

Cardium sp.

Acanthocardia paucicostata (Sowerby, 1841)

Rudicardium tuberculatum (Linné, 1758)

Superfamilia Veneroidea Rafinesque, 1815

Familia Veneridae Rafinesque, 1815

Chamalea gallina (Linné, 1758)

Callista sp.

Tapes sp.

CLASE GASTROPODA Cuvier, 1797

SUBCLASE PROSOBRANCHIA Milne Edwards, 1848 


\begin{tabular}{|c|c|c|c|c|c|c|c|}
\hline Listado especies/Estaciones & P. C. & P1 & $\mathbf{P 2}$ & P3 & P4 & P5 & Totales \\
\hline Striarca lactea & & & & 3 & & & 3 \\
\hline Glycymeris bimaculata & & & & 1 & & & 1 \\
\hline Glycymeris insubrica & 11 & 2 & 2 & 8 & & & 23 \\
\hline Glycymeris pilosa & 2 & & 3 & & & & 5 \\
\hline Glycymeris sp. & & 1 & & 10 & 1 & & 12 \\
\hline Aequipecten cf. opercularis & & & & & & 1 & 1 \\
\hline Pecten maximus & & 1 & & & & & 1 \\
\hline Neopycnodonte cochlear & & & & 1 & & & 1 \\
\hline Ctena decussata & & & & 2 & & & 2 \\
\hline Cardita calyculata & & & & 1 & & & 1 \\
\hline Cardium sp. & & 1 & & & & & 1 \\
\hline Acanthocardia paucicostata & 1 & & & 1 & 1 & & 3 \\
\hline Rudicardium tuberculatum & 2 & & 2 & 4 & 1 & & 9 \\
\hline Chamalea gallina & & 1 & & 4 & 1 & & 6 \\
\hline Callista $\mathrm{sp}$. & & & & 1 & & & 1 \\
\hline Tapes sp. & & & & 1 & & & 1 \\
\hline Diodora italica & & & & 1 & & & 1 \\
\hline Gibbula sp.1 & & & & 1 & & & 1 \\
\hline Gibbula sp.2 & & & & 1 & & & 1 \\
\hline Jujubinus striatus & & & & 2 & & & 2 \\
\hline Tricolia pullus pullus & & 2 & & 5 & & & 7 \\
\hline Cerithium vulgatum & & & & 1 & & & 1 \\
\hline Cerithium rupestre & & 2 & & 2 & & & 4 \\
\hline Bittium reticulatum & & 1 & & 4 & & & 5 \\
\hline Littorina neritoides & & & & 15 & & & 15 \\
\hline Turritella sp. & & 1 & & & & & 1 \\
\hline Rissoa variabilis & & 2 & & 5 & & & 7 \\
\hline Alvania pagodula & & & & 1 & & & 1 \\
\hline Hydrobia sp. & & 2 & & 8 & & & 10 \\
\hline Strombus bubonius & 2 & 1 & & 2 & 3 & & 8 \\
\hline Monophorus perversus & & & & 3 & & & 3 \\
\hline Murex sp. & & 2 & & & & & 2 \\
\hline Stramonita haemastoma & 1 & & & & & & 1 \\
\hline Gibberula miliaria & & & & 3 & & & 3 \\
\hline Gibberula sp. & & & & 1 & & & 1 \\
\hline Nassarius gibbosulus & & & & 1 & & & 1 \\
\hline Nassarius reticulatus & & & & 1 & & & 1 \\
\hline Nassarius sp.1 & & & & 1 & & & 1 \\
\hline Nassarius $\mathrm{sp} .2$ & & & & 1 & & & 1 \\
\hline Nassarius sp. 3 & & & & 2 & & & 2 \\
\hline Conus mediterraneus & & 1 & 1 & 1 & 1 & & 4 \\
\hline Bela nebula & & & & 2 & & & 2 \\
\hline Iberus alonensis & & & & 1 & & & 1 \\
\hline Scleractinia sp. & 1 & & & 1 & 1 & & 3 \\
\hline Echinidae sp. & & 1 & & 4 & & & 5 \\
\hline Totales & 20 & 21 & 8 & 107 & 9 & 1 & 166 \\
\hline
\end{tabular}

Tabla 1. Abundancias de las distintas especies para cada estación, sus cotas son las siguientes: P.C.: Peñón del Cuervo, cota 0,901,20 m s.n.m.; P1: Peñón del Cuervo2, cota 0,90-1,20 m s.n.m.; P2: Túnel de la antigua carretera del Peñón del Cuervo, cota 10-15 m s.n.m.; P3: Playa de la Araña, cota 0,90-1,20 m s.n.m.; P4: Punta Paloma, cota 0,90-1,20 m s.n.m.; P5: cota 30-35 m s.n.m.

Number of specimens of species by station. The levels are: P.C.: Peñón del Cuervo, 0.90-1.20 meters on sea level; P1: Peñón del Cuervo 2, 0.90-1.20 m on sea level; P2: Tonel of old road of Peñón del Cuervo, 10-15 m on sea level; P3: Araña beach, 0.90-1.20 m on sea level; P4: Punta Paloma, 0.90-1.20 m on sea level; P5: 30-35 m on sea level. 


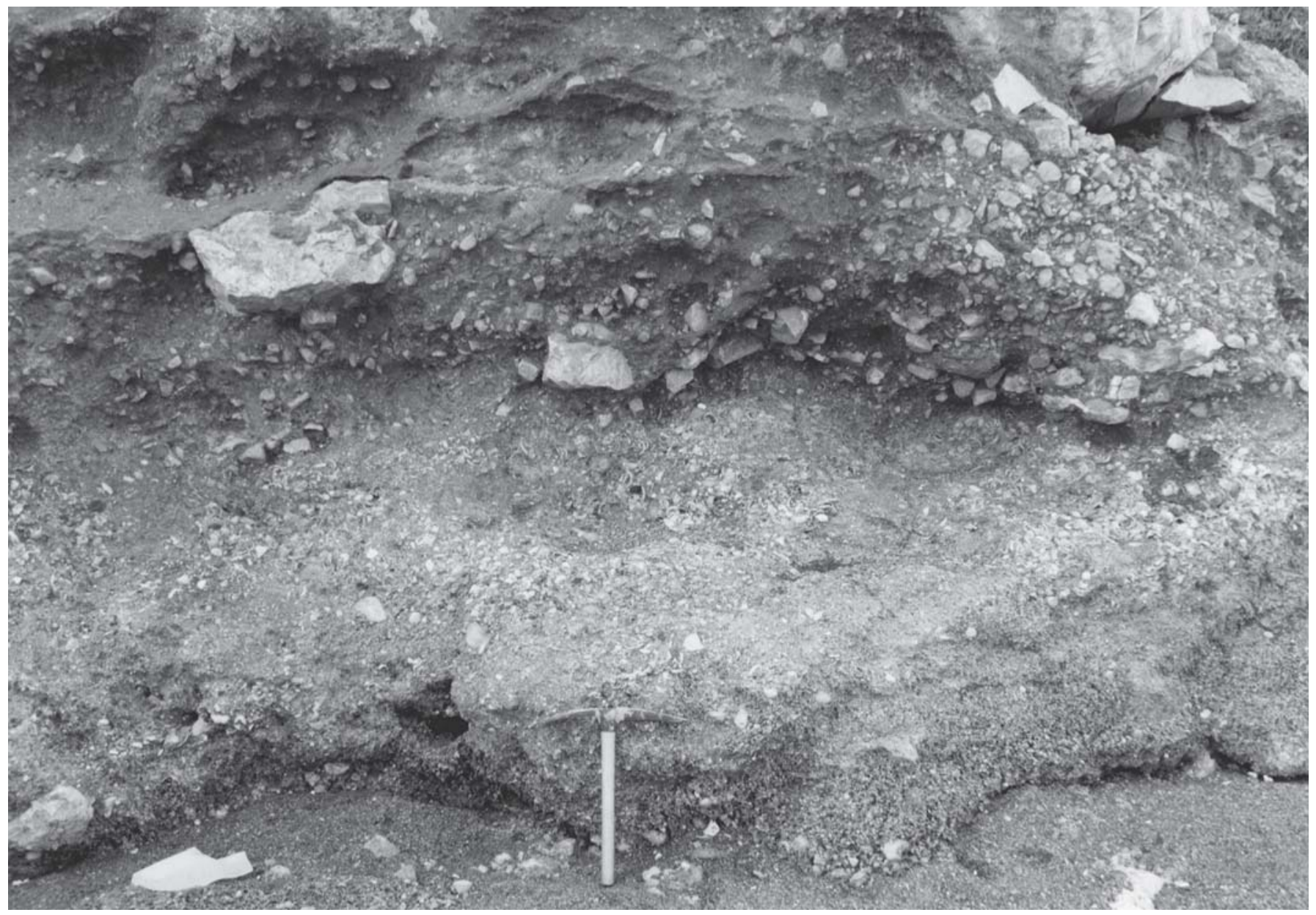

Figura 4. Estación P1 (Peñón del Cuervo2), donde se aprecian 2 estratos bien diferenciados: el estrato superior, continental con pulmonados [Iberus alonensis (Férussac, 1821)] y el inferior, Tirreniense con abundante malacofauna, cota 0,90-1,20 m s.n.m., debajo la playa actual.

P1 Station (Peñon del Cuervo 2), there are 2 different levels: the upper level is continental with helicid gastropods [Iberus alonensis (Férussac, 1821)] and the lower, Tyrrhenian with many molluscs to 0.90-1.20 m on sea level, under Holocene beach.

SUPERORDEN ARCHAEOGASTROPODA Thiele, 1925

ORDEN VETIGASTROPODA Salvini-Plawen \& Haszprunar, 1987

Superfamilia Fissurelloidea Fleming, 1822

Familia Fissurellidae Fleming, 1822

Diodora italica (Defrance, 1820)

Superfamilia Trochoidea Rafinesque, 1815

Familia Trochidae Rafinesque, 1815

Gibbula sp.1

Gibbula sp.2

Jujubinus striatus (Linné, 1758)

Familia Tricoliidae Robertson, 1985

Tricolia pullus pullus (Linné, 1758)

SUPERORDEN CAENOGASTROPODA Cox, 1959

ORDEN NEOTAENIOGLOSSA Haller, 1882

SUBORDEN DISCOPODA Fischer, 1884

Superfamilia Cerithioidea Férussac, 1819

Familia Cerithiidae Férussac, 1819
Cerithium (Thericium) vulgatum Bruguière, 1792

Cerithium (Thericium) rupestre Risso, 1826

Bittium reticulatum (Da Costa, 1778)

Superfamilia Littorinoidea Gray, 1840

Familia Littorinidae Gray, 1840

Littorina (Melaraphe) neritoides (Linné, 1758)

Familia Turritellidae Lovén, 1847

Turritella sp.

Superfamilia Rissoidea Gray, 1847

Familia Rissoidae Gray, 1847

Rissoa variabilis (von Muehlfeldt, 1824)

Alvania (Alvania) pagodula (Bucquoy, Dautzenberg \& Fischer, 1884)

Familia Hydrobiidae Troschel, 1857

Hydrobia sp.

Superfamilia Stromboidea Rafinesque, 1815

Familia Strombidae Rafinesque, 1815

Strombus (Strombus) bubonius (Defrance, 1825)

SUBORDEN PTENOGLOSSA Gray, 1853 


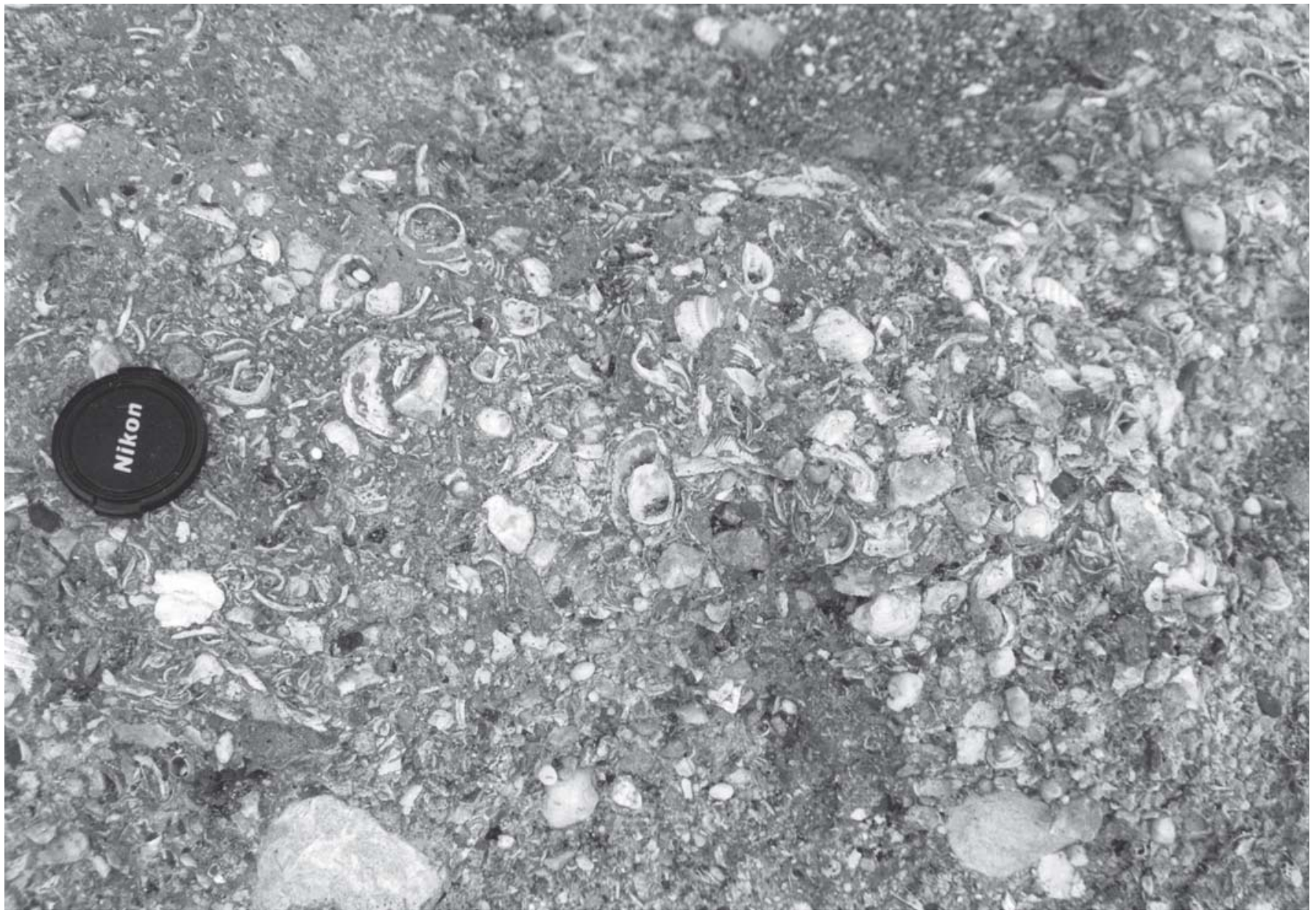

Figura 5. Detalle con abundantes valvas de Glycymeris sp. y Rudicardium tuberculatum (Linné, 1758) muy cementadas. Estación P1 (Peñón del Cuervo 2), cota 0,90-1,20 m s.n.m.

Level with Glycymeris sp. y Rudicardium tuberculatum (Linné, 1758) very compacted. Station P1 (Peñón del Cuervo 2), to 0.90-1.20 m on sea level.

Superfamilia Triphoroidea Gray, 1847

Familia Triphoridae Gray, 1847

Monophorus perversus (Linné, 1758)

ORDEN NEOGASTROPODA Thiele, 1929

Superfamilia Muricoidea Rafinesque, 1815

Familia Muricidae Rafinesque, 1815

Murex sp.

Stramonita haemastoma (Linné, 1758)

Familia Marginellidae Fleming, 1828

Gibberula miliaria (Linné, 1758)

Gibberula sp.

Familia Nassaridae Iredale, 1916

Nassarius gibbosulus (Linné, 1758)

Nassarius reticulatus (Linné, 1758)

Nassarius sp. 1

Nassarius sp.2

Nassarius sp. 3

Superfamilia Conoidea Rafinesque, 1815

Familia Conidae Rafinesque, 1815

Conus mediterraneus Hwass in Bruguière, 1792
Familia Turridae Swainson, 1840

Bela nebula (Montagu, 1803)

SUBCLASE PULMONATA Cuvier, 1817

ORDEN STYLOMMATOPHORA Schmidt, 1855

Superfamilia Helicoidea Rafinesque, 1815

Familia Helicidae Rafinesque, 1815

Iberus alonensis (Férussac, 1821)

Todas las especies obtenidas proceden de la zona infralitoral rocosa costera, excepto un fragmento encontrado del helícido Iberus alonensis, que se trata de un pulmonado que debe haber contaminado la muestra y que procede sin duda del estrato continental superior, muy rico en helícidos de esta especie, y separado por una clara discordancia.

\section{CONCLUSIONES}

Sólo en las cotas de 0,90-1,20 m s.n.m. en las estaciones o punto 11 (Peñón del Cuervo), punto 52 (Peñón del 
Cuervo), punto 48 (Playa de la Araña) y punto 46 (Punta Paloma), en la cota 10-15 ms.n.m, en la estación o punto 16 (túnel antigua carretera del Peñón del Cuervo) y 3035 m s.n.m. se ha recolectado fauna fósil correspondiente al Tirreniense por la presencia de Strombus bubonius y fauna asociada, correlacionable con el Tirreniense T-II (110.000-117.000 años) del nivel inferior del Faro de Roquetas de Mar (Almería) datación que se confirma con datos isotópicos (Zazo et al., 1981, 2003; Lario et al., 1999b) para la cota 0,90-1,20 m s.n.m. Para el caso de las cotas 10-11 y 30-35 m s.n.m. la fauna encontrada presenta una amplia distribución cronoestratigráfica: Glycymeris insubrica (Mioceno-Actualidad). En la cota 4-5 m s.n.m. había abundante fauna marina pero no fue muestreada por tratarse de un yacimiento arqueológico pendiente de conseguir los permisos de excavación.

En las cotas por encima de 10-11 m s.n.m. la fauna está casi ausente, por lo que el estudio paleontológico de estas cotas no ha podido corroborar los datos geológicos sobre estas playas fósiles o sobre su datación.

Debemos de considerar que las playas fósiles del Tirreniense del Peñón del Cuervo-La Araña-Rincón de la Victoria, son las segundas más importantes de todo el litoral andaluz por su valor paleontológico con una alta riqueza específica (46 especies identificadas) después de las de Almería.

Todas estas especies obtenidas proceden de la zona infralitoral rocosa costera, excepto el gasterópodo Iberus alonensis, pulmonado que procede del estrato continental superior, separado por una clara discordancia y de edad más moderna.

\section{AGRADECIMIENTOS}

Nuestro agradecimiento a todas aquellas personas que de un modo u otro han hecho posible realizar el presente estudio. Damos nuestro agradecimiento al Museo Municipal Paleontológico de Estepona (Ilmo. Ayuntamiento de Estepona) por facilitar el uso de sus dependencias para el presente estudio. El presente trabajo queda enmarcado dentro del Convenio entre la Universidad de Málaga y la Sociedad Financiera y Minera S.A. denominado "Inventario, Catalogación y Valoración de los Bienes Culturales (Arqueológicos, Geomorfológicos y Paisajísticos) en el territorio afectado por las canteras y la Fábrica de Cemento de la empresa Financiera y Minera S.A. en el paraje de la Araña”.

\section{BIBLIOGRAFÍA}

Azema, J. 1961. Etude geólogique des abords de Málaga (Espagne). Estudios Geológicos, 17, 131-160.
Blumenthal, M. 1927. Zum Bauplan betischer und penibetischer Decken in Norden der Provincz Málaga, Geological Rundschau, 17, 37-45.

Brükner, H. and Ratke, U. 1986. Paleoclimatic implication derived from profiles along the Spanish Mediterranean coast. In: Quaternari Climate in Westhern Mediterranea (Ed. F. López Vera), 467-486.

Durand-Delga, M. 1968. Coup d'oeil sur les unités Malaguides des Cordilléres Bétiques (Espagne). Comptes Rendus de la Academie de Sciences, 266, 190-193.

Durán, J. J. y Soria, J. M. 1989. II Encuentro de campo sobre Geomorfología, Cuaternario y Neotectónica. Instituto Geominero de España y AEQUA, Madrid, Libro-Guía, 42 pp.

Ferre, E. (Coord.) 2001. Inventario, catalogación y valoración de los bienes culturales (arqueológicos, morfológicos y paisajísticos) en el territorio afectado por las canteras y la fábrica de cemento de la empresa Financiera y Minera S.A. en el paraje de la Araña, Informe Técnico, 234 pp.

Lario, C. J., Zazo, C. y Goy, J. L. 1999a. El Cuaternario marino de Los Cantales. In: Patrimonio geológico de Andalucía. Ed. Enresa, Madrid, 283-285.

Lario, C.J., Zazo, C., Goy, J.L., Hoyos, M. y Hillaire Marcel, C. 1999b. Episodios marinos del último Interglacial (Estadio isotópico 5) del litoral de Málaga (SE penínsular). In: Elementos de los paisajes de la provincia de Málaga (coord. J.M. Senciales González y E. Ferre Bueno). Servicio de publicaciones, Universidad de Málaga, Málaga, 231-249.

Ramos, J. y Durán, J. J. 1998. El Solutrense de la Araña (Málaga). In: Las culturas del Pleistoceno superior de Andalucía (Eds. J.L. Sanchidrián y M.D. Simón). Patronato de la Cueva de Nerja, Nerja (Málaga), 63-75.

Sabelli, B., Giannuzzi-Savelli, R. i Bedulli, D. 1990. Catalogo annotato dei Molluschi marii del Mediterraneo, 1. Librería Naturafistica Bolognese, Bolonia, 348 pp.

Serrano, F., Sanz De Galdeano, C., Delgado, F., López, A.C. and Martin, A. 1995. The Mesozic and Cenozoic of the Malaguide Complex in the Málaga area: a Paleogene olistrostrome-type chaotic complex (Betic Cordillera, Spain). Geologie in Mijbouw, 74, 105-116.

Zazo, C., Goy, J.L., Hoyos, M., Dumas, B., Porta, J., Martinell, J., Baena, J. y Aguirre, E. 1981. Ensayo de síntesis sobre el Tirreniense peninsular español. Estudios Geológicos, 37, 257-262.

Zazo, C., Goy, J.L., Dabrio, C. J., Bardají, T., Hillaire-Marcel, C., Ghaleb, B., González Delgado, J. A. and Soles, V. 2003. Pleistocene raised marine terraces of the Spanish Mediterranean and Atlantic coasts: records of coastal uplift, sea-level highstands and climate changes. Marine Geology, 194, 103-133.

Manuscrito recibido: 19 de diciembre, 2002 Manuscrito aceptado: 6 de septiembre, 2003 